\title{
Rheological, texture, and sensory analyses and in vivo clinical efficacy of cosmetic formulations containing ascorbyl tetraisopalmitate
}

\author{
Análises reológica, de textura, e sensorial e eficácia clínica in vivo de formulações cosméticas \\ contendo tetraisopalmitato de ascorbila
}

\section{G. C. Bonilha, G. M. D. Costa, P. M. B. G. Maia Campos*}

School of Pharmaceutical Sciences of Ribeirão Preto - University of São Paulo, Av do Café, s/nº, Monte Alegre, Ribeirão Preto, SP, Brazil 14040-903

*Corresponding author: pmcampos@usp.br

\begin{abstract}
Cosmetics with anti-aging effects using ingredients of sustainable origin are increasing in the cosmetic market. This study aimed to evaluate the physical-mechanical and sensory properties and immediate clinical efficacy of cosmetic formulations with ingredients from natural sources to which were added or not (vehicle only) ascorbic acid (AAS) or ascorbyl tetraisopalmitate (ATIP). The preliminary stability, physical-mechanical properties, rheology texture profile, and sensory analysis were performed. In addition, immediate clinical effects in terms of skin hydration, transepidermal water loss (TEWL), and skin microrelief were investigated on the forearms and in the faces of nineteen subjects by noninvasive biophysical and skin imaging techniques. There were slight changes in the formulations with AAS submitted to thermal stress, but they were stable under ambient temperature. All formulations were effective in skin hydration. Serums and gel cream with ATIP decreased TEWL but did not significantly change the microrelief parameters. The serum achieved good sensory acceptance and parameters were correlated to those obtained by the texturometer. The best performance was from serum with ATIP, whereas gel cream was not well accepted. Therefore, results showed a correlation between physical-mechanical and sensorial properties, in addition to better stability and acceptance of the fat-soluble form of vitamin C.
\end{abstract}

Keywords: serum formulation, vitamin C, natural ingredients, clinical efficacy

\begin{abstract}
Resumo
Cosméticos antiaging com ingredientes naturais de origem sustentável são crescentes no mercado cosmético. Esse trabalho teve por objetivo avaliar as propriedades físico-mecânicas e sensoriais e a eficácia clínica imediata de formulações cosméticas à base de ingredientes de origem natural, nas formas de sérum e de gel creme, acrescidas ou não (veículo) de ácido ascórbico (AAS) ou tetraisopalmitato de ascorbila (TIPA) Foram avaliadas estabilidade preliminar, propriedades físico-mecânicas através de perfis de textura e reologia, análise sensorial e eficácia imediata nos antebraços e face de dezenove participantes, analisando hidratação, perda transepidérmica de água (TEWL) e microrrelevo cutâneo, por técnicas não invasivas incluindo a imagem da pele. Houve leves alterações nas formulações com AAS submetidas ao estresse térmico, sendo estáveis sob temperatura ambiente. As formulações foram eficazes na hidratação da pele e os séruns e gel creme que continham TIPA diminuíram a TEWL, mas não alteraram significativamente os parâmetros do microrrelevo. O sérum obteve boa aceitação sensorial, com parâmetros correlacionados aos obtidos pelo texturômetro, sendo o sérum com TIPA melhor avaliado, enquanto que o gel creme não foi bem aceito. Portanto, os resultados obtidos mostraram relação entre características físico-mecânicas e sensoriais, além da melhor estabilidade e aceitação da forma lipossolúvel da vitamina $\mathrm{C}$.
\end{abstract}

Palavras-chave: sérum formulation, vitamina $\mathrm{C}$, ingredientes de origem natural, eficácia clínica 


\section{Introduction}

Antiaging, minimizing skin changes caused by aging or photoaging, is one of the most desired characteristics for some cosmetic consumers. Products claiming antiaging effects intend to act in the improvement of skin hydration and skin pigmentation pattern while potentially reducing expression lines. Antioxidants compounds have been added to cosmetic antiaging formulations due to their reductive properties, neutralizing reactive oxygen species and minimizing the damage that could be caused to DNA and protein molecules (1).

Among the main antioxidants used is ascorbic acid or vitamin $\mathrm{C}$, which also acts as an essential cofactor in the enzymatic reaction of collagen synthesis, and improves overall skin appearance (2-5). However, ascorbic acid can exhibit instability in the presence of oxygen, high temperatures, light, and high $\mathrm{pH}$. Formulations containing ascorbic acid may lose their antioxidant potential, as well as undergo visible changes over time, especially in colour $(2,6)$.

Because of their higher stability, some formulations have different forms of vitamin $\mathrm{C}$, such as the fat-soluble derivative ascorbyl tetraisopalmitate, whose applications and stability has been described in studies and has shown proven efficacy as a precursor of vitamin $\mathrm{C}$, offering the same positive effects and other benefits related to penetration and hydration (7). However, in order to increase the production of collagen, it is necessary to have the vitamin in its free form. Therefore, there must be a mechanism of hydrolysis of the compound after penetration, which may imply a partial use of the substance, as the conversion might not be sufficiently effective.

There has been a search for more natural and biocompatible products in the cosmetic market, those which are composed of ingredients of natural or sustainable origin and present a pleasant cosmetic form, since the sensory perception influences on the purchase and use (8). In this context, serums have had high adhesion by the consumer due to their practical application, as they usually have good spreadability, fast absorption, and low stickiness (9). This study aimed to evaluate the physical-mechanical and sensory properties and immediate clinical efficacy of cosmetic formulations based on ingredients from natural sources in the form of serum and gel cream to which were added or not (vehicle only) ascorbic acid (AAS) or ascorbyl tetraisopalmitate (ATIP).

\section{Introdução}

Antienvelhecimento é um dos apelos mais desejados para o consumidor de cosméticos, o qual consiste em minimizar as alterações da pele causadas pelo envelhecimento ou fotoenvelhecimento. Os produtos que reivindicam efeitos antienvelhecimento visam atuar na melhoria do padrão de hidratação e pigmentação da pele, enquanto podem reduzir as linhas de expressão. Os antioxidantes são compostos que vem sendo muito adicionados em formulações cosméticas antiaging em função das suas propriedades redutoras, neutralizando as espécies reativas de oxigênio e minimizando os danos que poderiam ser causados às moléculas de DNA e proteínas (1).

Dentre os principais antioxidantes adicionados está o ácido ascórbico ou vitamina $\mathrm{C}$, que também age como cofator essencial na reação enzimática de síntese de colágeno, promovendo a melhora da aparência geral da pele (2-5). No entanto, pode apresentar instabilidade na presença de oxigênio, altas temperaturas, luz e $\mathrm{pH}$ elevado, e as formulações que o contêm podem perder seu potencial antioxidante, assim como sofrer alterações visíveis ao longo do tempo, especialmente na cor $(2,6)$. Algumas formulações têm formas derivadas da vitamina $\mathrm{C}$, por apresentarem maior estabilidade, como o derivado lipossolúvel tetraisopalmitato de ascorbila, que já teve suas aplicações e estabilidade descritas em estudos, mostrando eficácia comprovada como um precursor da vitamina $\mathrm{C}$, com os efeitos dessa e outros benefícios relacionados à penetração e hidratação (7). Entretanto, para que haja aumento da produção de colágeno, é necessária a presença da vitamina na forma livre. Por isso, deve haver um mecanismo de hidrólise do composto após a penetração, o que pode implicar em um aproveitamento parcial da substância, uma vez que a conversão pode não ser efetiva o suficiente.

No mercado cosmético há uma busca por produtos mais naturais e biocompatíveis, os quais são compostos por ingredientes de origem natural ou sustentável, e com forma cosmética agradável, uma vez que a percepção sensorial influencia na compra e uso (8). Nesse contexto, os séruns têm tido alta aderência pelo consumidor, devido à praticidade de aplicação, uma vez que apresentam boa espalhabilidade, rápida absorção e baixa pegajosidade (9). Este estudo teve por objetivo avaliar as propriedades físico-mecânicas e sensoriais e a eficácia clínica imediata de formulações cosméticas à base de ngredientes de origem natural, nas formas de sérum e de gel creme, adicionadas ou não (veículo) de ácido ascórbico (AAS) ou tetraisopalmitato de ascorbila (TIPA). 


\section{Material and Methods}

The studied formulations are described in the Table 1. More than $97 \%$ of the composition of the developed vehicles were ingredients of natural or sustainable origin. The gel cream (GC) and serum (SE) cosmetic forms had the same aqueous phase and thus mostly the same composition. Formulations were evaluated as vehicle (without active ingredients) and with ATIP or with AAS, incorporated at $2 \%$.

\section{Formulations' characterization and preliminary stability tests}

Formulations were submitted to centrifugation using a centrifuge test (CentiBio ${ }^{\circledR}$ 80-2B, Biovera, Brazil) to evaluate the occurrence of phase separation. Rheological analysis of formulations was performed using a cone and plate type digital rheometer (DV-III ${ }^{\circledR}, \mathrm{AM}-$ ETEK Brookfield Middleboro, MA, USA), with 0.500 $\mathrm{g}$ of formulation in each analysis, obtaining apparent minimum viscosity, flow and consistency indexes, and thixotropy values. The texture profile was studied from spreadability, texture and hardness tests obtained with a texturometer (TA.XT/Plus ${ }^{\circledR} 50$, Extralab, Itatiba, SP,

\section{Material e Métodos}

As formulações estudadas são descritas na Tabela 1, sendo que mais de $97 \%$ da composição dos veículos desenvolvidos eram ingredientes de origem natural ou sustentável. As formas cosméticas gel creme (GC) e sérum (SE) tiveram a mesma fase aquosa e, assim, a maior parte da composição em comum. As formulações foram avaliadas na forma de veículo (sem ingredientes ativos) e com TIPA ou AAS, incorporados a $2 \%$.

Caracterização e testes de estabilidade preliminar das formulações

As formulações foram submetidas a um teste de centrifugação em centrífuga (CentiBio ${ }^{\circledR}$ 80-2B, BioVera, Brasil) para avaliar ocorrência de separação de fases. A análise reológica das formulações foi realizada no digital reômetro tipo cone e placa (DV-III ${ }^{\circledR}$ AMETEK Brookfield, Middleboro, MA, EUA), com 0,500 g de formulação em cada análise, obtendo valores de viscosidade aparente, índices de fluxo e de consistência e tixotropia. O perfil de textura foi estudado com testes de espalhabilidade, textura e dureza, feitos no texturômetro (TA.XT/Plus ${ }^{\circledR} 50$, Extralab, Itatiba, SP, Brasil), que

Table 1 - Composition of studied formulations: GC, GC-AAS, GC-ATIP, SE, SE-AAS and SE-ATIP Tabela 1 - Composição das formulações estudadas: GC, GC-AAS, GC-TIPA, SE, SE-AAS e SE-TIPA

\begin{tabular}{|c|c|c|c|c|c|c|c|c|c|c|c|c|c|c|}
\hline $\begin{array}{l}\text { Composition/ } \\
\text { Composição } \\
\text { (INCI Name) }\end{array}$ & $\begin{array}{l}1 \\
1 \\
1 \\
1 \\
1\end{array}$ & $\begin{array}{l}\text { Supplier/ } \\
\text { Fornecedor }\end{array}$ & $\begin{array}{l}1 \\
1 \\
1 \\
1 \\
1\end{array}$ & GC & $\begin{array}{l}1 \\
1 \\
1\end{array}$ & GC-AAS & & $\begin{array}{r}\text { Form } \\
\text { Form } \\
\text { GC-ATIP } \\
\text { GC-TIPA } \\
\end{array}$ & $\begin{array}{l}\text { Iul } \\
1 \\
1\end{array}$ & $\begin{array}{l}\overline{\text { ons/ }} \\
\text { ōes }\end{array}$ & $\begin{array}{l}1 \\
1 \\
1\end{array}$ & SE-AAS & $\begin{array}{l}1 \\
\text { I }\end{array}$ & $\begin{array}{c}\text { SE-ATIP / } \\
\text { SE-TIPA }\end{array}$ \\
\hline Hydroxyethylcellulose & 1 & Galena $^{\circledR}$ & 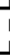 & $\checkmark$ & $\begin{array}{l}T \\
1\end{array}$ & $\checkmark$ & i & $\checkmark$ & 1 & $\checkmark$ & T & $\checkmark$ & T & $\checkmark$ \\
\hline Xanthan gum & 1 & $\mathrm{CP} \mathrm{Kelco}^{\circledR}$ & 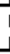 & $\checkmark$ & i & $\checkmark$ & T & $\checkmark$ & i & $\checkmark$ & I & $\checkmark$ & i & $\checkmark$ \\
\hline Disodium EDTA & I & Mapric $^{\circledR}$ & 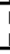 & $\checkmark$ & i & $\checkmark$ & i & $\checkmark$ & i & $\checkmark$ & i & $\checkmark$ & i & $\checkmark$ \\
\hline Sodium metabisulfite & i & Mapric $^{\circledR}$ & 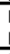 & $\checkmark$ & i & $\checkmark$ & i & $\checkmark$ & i & $\checkmark$ & i & $\checkmark$ & i & $\checkmark$ \\
\hline Propylene glycol & i & Nikkol $^{\circledR} /$ Galena $^{\circledR}$ & 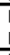 & $\checkmark$ & 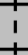 & $\checkmark$ & i & $\checkmark$ & i & $\checkmark$ & 1 & $\checkmark$ & I & $\checkmark$ \\
\hline Glycerin & i & Mapric $^{\circledR}$ & 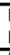 & $\checkmark$ & 1 & $\checkmark$ & i & $\checkmark$ & I & $\checkmark$ & I & $\checkmark$ & I & $\checkmark$ \\
\hline C13-15 Alkane & T & Neossance $^{\circledR} /$ Galena $^{\circledR}$ & 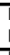 & $\checkmark$ & 1 & $\checkmark$ & i & $\checkmark$ & I & & 1 & & I & \\
\hline Steareth-2 & T & Croda $^{\circledR}$ & 7 & $\checkmark$ & 1 & $\checkmark$ & I & $\checkmark$ & 1 & & 1 & & 1 & \\
\hline Steareth-21 & 1 & $\mathrm{Croda}^{\circledR}$ & 7 & $\checkmark$ & 1 & $\checkmark$ & 1 & $\checkmark$ & 1 & & 1 & & 1 & \\
\hline $\begin{array}{c}\text { Butylated } \\
\text { hydroxytoluene }\end{array}$ & $\begin{array}{l}T \\
\text { I } \\
\text { I }\end{array}$ & Mapric $^{\circledR}$ & 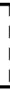 & $\checkmark$ & $\begin{array}{l}1 \\
1 \\
1 \\
1\end{array}$ & $\checkmark$ & i & $\checkmark$ & 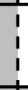 & & $\begin{array}{l}1 \\
1 \\
1 \\
\end{array}$ & & $\begin{array}{l}1 \\
1 \\
1\end{array}$ & \\
\hline Xylityl Sesquicaprylate & i & Chemyunion $^{\circledR}$ & T & $\checkmark$ & 1 & $\checkmark$ & i & $\checkmark$ & I & $\checkmark$ & I & $\checkmark$ & I & $\checkmark$ \\
\hline Aqua & 1 & - & 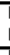 & $\checkmark$ & 1 & $\checkmark$ & i & $\checkmark$ & i & $\checkmark$ & 1 & $\checkmark$ & 1 & $\checkmark$ \\
\hline $\begin{array}{c}\text { Polyglyceryl-10 } \\
\text { Myristate, } \\
\text { Triethylhexanoin, } \\
\text { Glycerin, Water }\end{array}$ & $\begin{array}{l}1 \\
1 \\
1 \\
1 \\
1 \\
1 \\
1\end{array}$ & Nikkol $^{\circledR} /$ Galena $^{\circledR}$ & 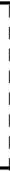 & $\checkmark$ & $\begin{array}{l}\text { i } \\
\text { i } \\
\text { i } \\
\text { i } \\
\text { i }\end{array}$ & $\checkmark$ & i & $\checkmark$ & $\begin{array}{l}\text { i } \\
\text { i } \\
\text { i } \\
\text { i } \\
\text { i }\end{array}$ & $\checkmark$ & $\begin{array}{l}1 \\
1 \\
1 \\
1 \\
i \\
i\end{array}$ & $\checkmark$ & i & $\checkmark$ \\
\hline $\begin{array}{c}\text { Ascorbyl } \\
\text { Tetraisopalmitate }\end{array}$ & i & Nikkol $^{\circledR} /$ Galena $^{\circledR}$ & 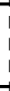 & & $\begin{array}{l}T \\
1 \\
1 \\
\end{array}$ & & $\begin{array}{l}T \\
1 \\
1 \\
\end{array}$ & $\checkmark$ & $\begin{array}{l}1 \\
1 \\
1 \\
\end{array}$ & & $\begin{array}{l}1 \\
1 \\
1 \\
1\end{array}$ & & $\begin{array}{l}1 \\
1 \\
1 \\
\end{array}$ & $\checkmark$ \\
\hline Ascorbic acid & I & Galena $^{\circledR}$ & 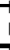 & & 1 & $\checkmark$ & 1 & & 1 & & 1 & $\checkmark$ & 1 & \\
\hline
\end{tabular}


Brazil), which provides time-dependent power graphs (10). The spreadability test ("TTC Spreadability Rig") provided results of firmness and work of shear, the texture test (A/BE-d45 "Back extrusion Rig $45 \mathrm{~mm}$ DISC") indicated parameters of firmness, consistency, cohesiveness and viscosity index, and the hardness test (" $\mathrm{P} / 45 \mathrm{C}$ " Penetration conical probe) resulted in hardness, cohesiveness and stickiness values.

The formulations were GC (vehicle gel cream), GCAAS (gel cream with AAS), GC-ATIP (gel cream with ATIP), SE (vehicle serum), SE-AAS (serum with AAS) and SE-ATIP (serum with ATIP). These were submitted to a preliminary stability study, stored at temperatures of $25^{\circ} \mathrm{C}$ and $45^{\circ} \mathrm{C}$, and measuring $\mathrm{pH}$ (Tecnal ${ }^{\circledR}$ TEC$3 \mathrm{MPp}$, Tecnopon, Piracicaba SP, Brazil) and rheology, as well as visually evaluating organoleptic characteristics (appearance, colour, odour), for 14 days.

\section{Study Participants}

Nineteen female subjects, aged from 39 to 55 years (medium of $46.3 \pm 4.6$ years old), Fitzpatrick skin phototype II and III, were enrolled and signed a free and informed consent form. The study was approved by the Ethics Committee of the Faculty of Pharmaceutical Sciences - University of Sao Paulo (CAAE: 84599418.8.0000.5403). The subjects were divided into three balanced groups with average baseline hydration measurements in the malar region of the face approximately equal between them.

\section{Methodology}

Sensory analysis and immediate efficacy tests were performed in the clinical studies. In the sensory analysis, seven randomized areas of $4 \mathrm{~cm}^{2}$ of the inner part of the forearms were studied for all formulations (GC, GCAAS, GC-ATIP, SE, SE-AAS and SE-ATIP) according to the participants' perception after the application (20 $\mu \mathrm{L}$ ) of formulations in each determined region of the forearm, maintaining one clean for control. Questionnaires were graded from one to five (bad, regular, good, very good and excellent, respectively) and applied to assess the moisture and soft skin feeling, spreadability, stickiness, skin touch (immediately after application and after five minutes), and purchase intention.

For the efficacy tests, each group received the application of one of the three serums (SE, SE-AAS or SEATIP) on the entire face ( $1 \mathrm{~g}$ of formulation). Evaluation took place the malar region application, before and two hours after application, as well as all areas and on the forearm in those areas used for sensory analysis. The stratum corneum water content was measured in five replicates (Corneometer $^{(B)}$ TMCM 825, Courage fornece gráficos de força em função do tempo (10). O teste de espalhabilidade ("TTC Spreadability Rig") forneceu resultados de firmeza e trabalho de cisalhamento, o teste de textura (A/BE-d45 "Back extrusion Rig 45 mm DISC"), indicou parâmetros de firmeza, consistência, coesividade e índice de viscosidade, e o teste de dureza (probe cônico de penetração "P/45C") resultou em valores de dureza, coesividade e pegajosidade.

As formulações GC (gel creme veículo), GC-AAS (gel creme com AAS), GC-TIPA (gel creme com TIPA), SE (sérum veículo), SE-AAS (sérum com AAS) e SE-TIPA (sérum com TIPA) foram submetidas a estudo de estabilidade preliminar, armazenadas em temperatura de 25 ${ }^{\circ} \mathrm{C}$ e em estufa à $45^{\circ} \mathrm{C}$, sendo registrados valores de $\mathrm{pH}$ (Tecnal ${ }^{\circledR}$ TEC-3MPp, Tecnopon, Piracicaba SP, Brazil) e reologia por 14 dias, além da avaliação visual de características organolépticas (aspecto, cor, odor).

\section{Participantes do estudo}

Foram recrutadas dezenove participantes de pesquisa do sexo feminino, faixa etária de 39 a 55 anos (média de 46,3 \pm 4,6 anos) e fototipos de Fitzpatrick II e III, que assinaram termo de consentimento livre e esclarecido. O estudo foi aprovado pelo Comitê de Ética em Pesquisa Clínica da Faculdade de Ciências Farmacêuticas da Universidade de São Paulo (CAAE: 84599418.8.0000.5403). As participantes foram divididas em três grupos equilibrados com medidas basais médias de hidratação na região malar do rosto aproximadamente iguais entre eles.

\section{Metodologia}

Nos estudos clínicos, foram realizadas análises sensoriais e testes imediatos de eficácia. Na avaliação sensorial, foram estudadas sete áreas randomizadas de $4 \mathrm{~cm}^{2}$ da parte interna dos antebraços para todas as formulações (GC, GC-AAS, GC-TIPA, SE, SE-AAS e SE-TIPA) de acordo com a percepção das participantes após a aplicação $(20 \mu \mathrm{L})$ das formulações em cada região demarcada do antebraço, mantendo uma limpa para controle. Questionários foram classificados de um a cinco (ruim, regular, bom, muito bom e ótimo, respectivamente) e aplicados para avaliar a sensação de hidratação e maciez da pele, espalhabilidade, pegajosidade, sensação ao toque imediata e após cinco minutos, e intenção de compra.

Para os testes de eficácia, cada grupo recebeu a aplicação de um dos três séruns (SE, SE-AAS ou SE-TIPA) no rosto todo ( $1 \mathrm{~g}$ de formulação) e a avaliação foi feita na região malar, antes e após duas horas da aplicação, nas mesmas áreas do antebraço usadas para a análise sensorial. O conteúdo aquoso do estrato córneo foi me- 
\& Khazaka, Cologne, FRD) and TEWL values were obtained in triplicate (Tewameter ${ }^{\circledR}$ TM210, Courage \& Khazaka, Cologne, FRD). Skin microrelief images were recorded (VisioScan ${ }^{\circledR}$ VC98, Courage \& Kazaka, Cologne, FRD) and analysed for parameters which describe the skin surface (SELS - Surface Evaluation of Living Skin): SEr (roughness), SEsc (desquamation), SEw (number and width of wrinkles) and SEsm (smoothness).

\section{Data analysis and Statistics}

All statistical analysis had a 95\% confidence interval and the data sets were analysed for normality before defining which statistical tests were applicable in each case. For rheology, texture, and $\mathrm{pH}$ data, unidirectional and Tukey analysis of variance tests, or Kruskal-Wallis non-parametric followed by Dunns, were applied. For clinical study data sets, the t-test was used for parametric and the Mann-Whitney test for non-parametric analysis.

\section{Results and Discussion}

In the preliminary centrifugation test, all formulations were unchanged. GC and SE vehicle formulations, and these added with ATIP, had an initial mean $\mathrm{pH}$ of 5.4, whereas those containing AAS had $\mathrm{pH}$ values of 3.3, on average, due to the acidic characteristic of the active substance.

The decision was made to not adjust the $\mathrm{pH}$ of the AAS formulations to avoid instability seen in preliminary tests with $\mathrm{pH}$ adjustments made. Thus, the formulation was recommended for night use only.

At rheological characterization, the shear stress curves (Figure 1) showed higher shear stress for the GC formulations, indicating higher viscosities than those of the serums. This behaviour was confirmed by minimum apparent viscosity and by the texture test results, in which the parameters of viscosity index and cohesiveness were higher for GC and GC-AAS, intermediate for GC-ATIP, and the lowest were found for the serums, with values very close to each other. In addition, both the consistency index from rheology and the firmness and consistency from texture test indicated higher values for the gel cream comparing to the serum. Hysteresis areas were the same and quite low, indicating the polymers' intrinsic characteristic of low thixotropy, since both xanthan gum and hydroxyethyl cellulose have pseudoplastic behaviour, with great ease of viscosity recovery once shear stress is no longer applied $(11,12)$.

In the stability study by thermal stress, formulations dido (Corneometer ${ }^{\circledR}$ TMCM 825, Courage \& Khazaka, Cologne, RFA), em cinco réplicas, e valores de TEWL foram obtidos (Tewameter ${ }^{\circledR}$ TM210, Courage \& Khaza$\mathrm{ka}$, Cologne, RFA) em triplicata. As imagens de microrrelevo cutâneo foram registradas (VisioScan ${ }^{\circledR}$ VC98, Courage \& Khazaka, Cologne, RFA), e analisadas com relação aos parâmetros que descrevem a superfície da pele (SELS - Surface Evaluation of Living Skin): SEr (rugosidade), SEsc (descamação), SEw (número e largura das rugas) e SEsm (suavidade).

\section{Análise de dados e Estatística}

Todas as análises estatísticas tiveram 95\% de intervalo de confiança e os conjuntos de dados foram analisados com relação à normalidade antes de definir quais os testes estatísticos aplicáveis em cada caso. Para dados de reologia, textura e $\mathrm{pH}$, foram aplicados os testes de análise de variância unidirecional e Tukey, ou o nãoparamétrico de Kruskal-Wallis seguido de Dunns. Em dados do estudo clínico, foi utilizada a análise do t-test para dados paramétricos, e Mann-Whitney test para dados não-paramétricos.

\section{Resultados e Discussão}

Nos testes de estabilidade preliminar por centrifugação todas as formulações se mostraram inalteradas. As formulações GC e SE veículos, e adicionadas de TIPA, apresentaram $\mathrm{pH}$ inicial de 5,4, em média, já as que continham AAS tiveram valores de $\mathrm{pH}$ de 3,3, em média, devido à característica ácida da substância ativa. Optou-se por não corrigir o pH das formulações de AAS para evitar a instabilidade vista em testes preliminares com correção. Desta forma, a formulação foi recomendada apenas para uso noturno.

Na caracterização de reologia, as curvas de tensão de cisalhamento (Figura 1) mostraram maior tensão para cisalhar as formulações GC, com viscosidades superiores às dos séruns, comportamento confirmado pelos resultados de viscosidade mínima aparente, e pelo teste de textura, com os parâmetros de índice de viscosidade e de coesividade, em que GC e GC-AAS possuíram os maiores valores, GC-TIPA, se mostrou intermediário, e os séruns tiveram os menores, com valores bastante próximos. Além disso, tanto o índice de consistência da reologia, quanto a firmeza e consistência do teste de textura, apontaram valores superiores para o gel creme em relação ao sérum. As áreas de histerese foram bastante baixas e iguais, indicando a característica intrínseca dos polímeros de apresentarem baixa tixotropia, uma vez que tanto a goma xantana, quanto a hidroxietilcelulose, têm comportamento pseudoplástico, com 


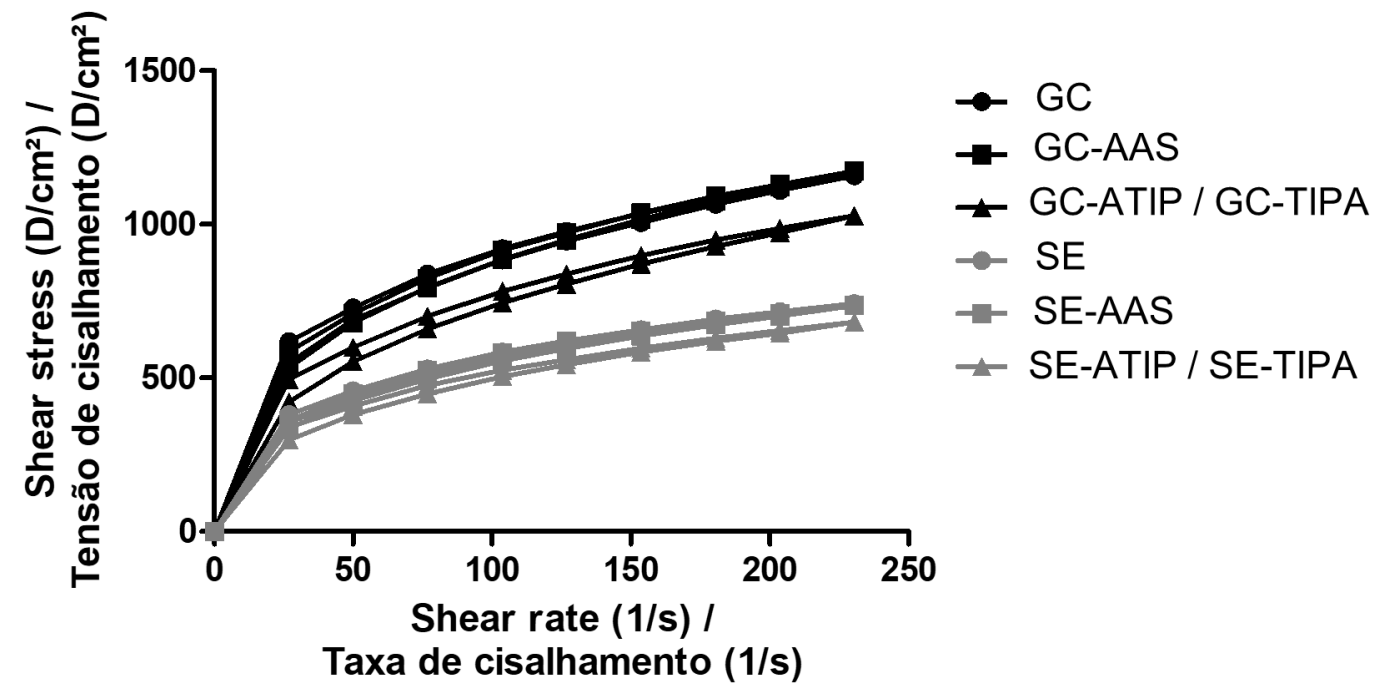

Figure 1 - Shear stress curves as a function of shear rate of the studied formulations

Figura 2 - Curvas de tensão de cisalhamento em função de taxa de cisalhamento das formulações estudadas

Table 2 - Measured pH values in the preliminary stability study of the GC, GC-AAS, GC-ATIP, SE, SE-AAS and SE-ATIP formulations

Tabela 2 - Valores medidos de $\mathrm{pH}$ ao longo do estudo de establidade preliminar das formulações GC, GC-AAS, GC-TIPA, SE, SE-AAS e SE-TIPA

\begin{tabular}{|l|cccccc|}
\cline { 2 - 7 } \multicolumn{1}{c|}{} & GC & GC-AAS & $\begin{array}{c}\text { GC-ATIP } / \\
\text { GC-TIPA }\end{array}$ & SE & SE-AAS & $\begin{array}{c}\text { SE-ATIP } \\
\text { SE-TIPA }\end{array}$ \\
\hline \multirow{3}{*}{ T0 } & 5.47 & 3.37 & 5.19 & 5.38 & 3.24 & 5.52 \\
& 5.43 & 3.34 & 5.22 & 5.39 & 3.25 & 5.47 \\
& 5.41 & 3.31 & 5.22 & 5.38 & 3.26 & 5.45 \\
\hline \multirow{3}{*}{ T7 - $25^{\circ} \mathrm{C}$} & 5.29 & - & 5.26 & 5.21 & - & 5.43 \\
& 5.33 & - & 5.23 & 5.27 & - & 5.40 \\
& 5.32 & - & 5.24 & 5.28 & - & 5.43 \\
\hline \multirow{3}{*}{$\mathrm{T} 7-45^{\circ} \mathrm{C}$} & 5.41 & - & 5.28 & 5.24 & - & 5.34 \\
& 5.37 & - & 5.29 & 5.23 & - & 5.34 \\
& 5.32 & - & 5.29 & 5.25 & - & 5.30 \\
\hline \multirow{3}{*}{ T14 - $25^{\circ} \mathrm{C}$} & 5.42 & 3.33 & 5.23 & 5.27 & 3.32 & 5.32 \\
& 5.45 & 3.33 & 5.22 & 5.24 & 3.32 & 5.31 \\
& 5.32 & 3.34 & 5.23 & 5.24 & 3.32 & 5.30 \\
\hline \multirow{3}{*}{$\mathrm{T} 14-45^{\circ} \mathrm{C}$} & 5.29 & 3.31 & 5.41 & 5.20 & 3.27 & 5.17 \\
& 5.23 & 3.31 & 5.30 & 5.18 & 3.27 & 5.14 \\
& 5.17 & 3.32 & 5.32 & 5.17 & 3.29 & 5.16 \\
\hline
\end{tabular}



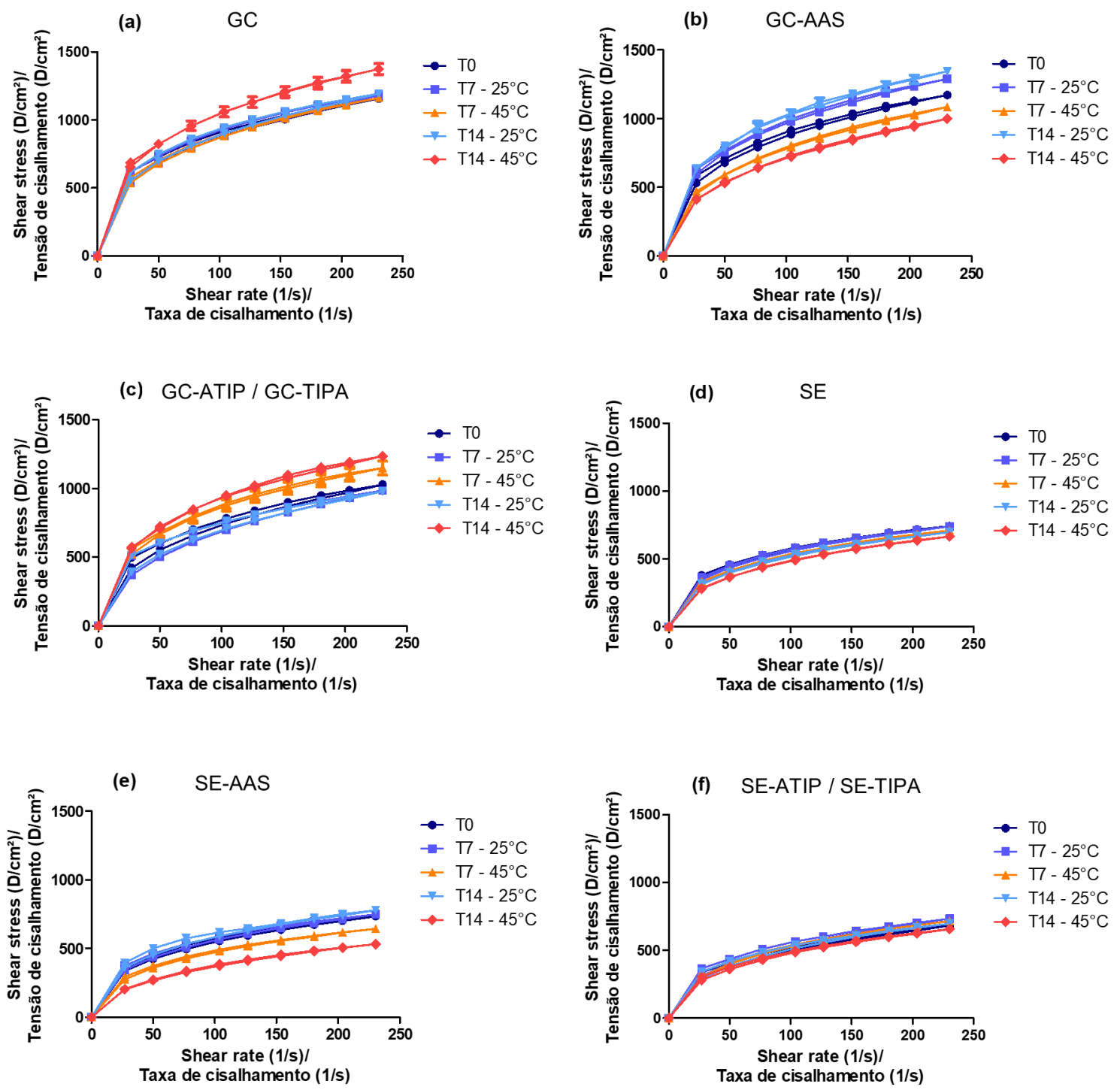

Figure 2 - Comparative results of shear stress curves versus shear rate during the preliminary stability study of formulations GC (a), GC-AAS (b), GC-ATIP (c), SE (d), SE-AAS (e) and SE- ATIP (f)

Figura 2 - Resultados comparativos das curvas de tensão de cisalhamento em função de taxa de cisalhamento ao longo do estudo de estabilidade preliminar das formulações GC (a), GC-AAS (b), GC-TIPA (c), SE (d), SE-AAS (e) e SE-TIPA (f)

those containing AAS showed a slight colour change after 14 days, likely due to an initiation of the oxidation process, and most showed an expected decrease in $\mathrm{pH}$ (Table 2), justified by the high aqueous content and the influence of temperature on $\mathrm{pH}$ (13). The apparent minimum viscosity and consistency index both decreased and there was an increase in flow index in the AAS formulations under thermal stress, confirming instability only under this condition (Figure 2). The SE and SE-ATIP formulations presented higher stability, and a similar result was obtained in a study of formulations containing ATIP, in which vehicle and formulation with active ingredient did not differ from each other in terms grande facilidade de recuperação da viscosidade, uma vez que a tensão de cisalhamento para de ser aplicada $(11,12)$.

No estudo de estabilidade por estresse térmico, as formulações que continham AAS apresentaram leve alteração de cor após 14 dias, devido a um provável início de oxidação, e a maior parte delas apresentou diminuição esperada do $\mathrm{pH}$ (Tabela 2), justificada pelo alto conteúdo aquoso e a influência da temperatura no $\mathrm{pH}$ (13). A viscosidade mínima aparente e índice de consistência diminuíram e houve aumento do índice de fluxo nas formulações de AAS em estresse térmico, confirmando instabilidade apenas nessa condição 
of viscosity and consistency index parameters, which were kept stable through the preliminary study period with storage at temperatures of $25^{\circ} \mathrm{C}$ and $45^{\circ} \mathrm{C}$ (14).

The serum formulations were well accepted, as shown in Figure 3, which stood out regarding great spreadability, skin touch, and hydration, with purchase intentions of $37 \%$ for SE, $37 \%$ for SE-AAS and $63 \%$ for SE-ATIP. The texturometer spreadability results confirmed its sensory predictive nature, since SE and SE-ATIP presented lower work of shear that would indicate better spreadability, according to the verified correlation between both parameters $(10,15)$. On the other hand, the GC formulations did not have good acceptance, with purchase intention between 5 and $11 \%$. In the hardness test, these formulations presented
(Figura 2). As formulações SE e SE-TIPA apresentaram maior estabilidade, e um resultado similar foi obtido em estudo de formulações contendo TIPA, em que veículo e formulação com ingrediente ativo não se diferenciaram com relação aos parâmetros de viscosidade e índice de consistência, além de terem se mantido estáveis durante estudo preliminar com armazenamentos sob temperaturas $25^{\circ} \mathrm{C}$ e $45^{\circ} \mathrm{C}$ (14).

Houve boa aceitação das formulações de sérum, como apresentado na Figura 3, que se destacaram com relação à ótima espalhabilidade, sensação ao toque e hidratação, com intenção de compra de $37 \%$ para SE, $37 \%$ para SE-AAS e $63 \%$ para SE-TIPA. Os resultados de espalhabilidade do texturômetro confirmaram o carácter preditivo de sensorial do equipamento, uma

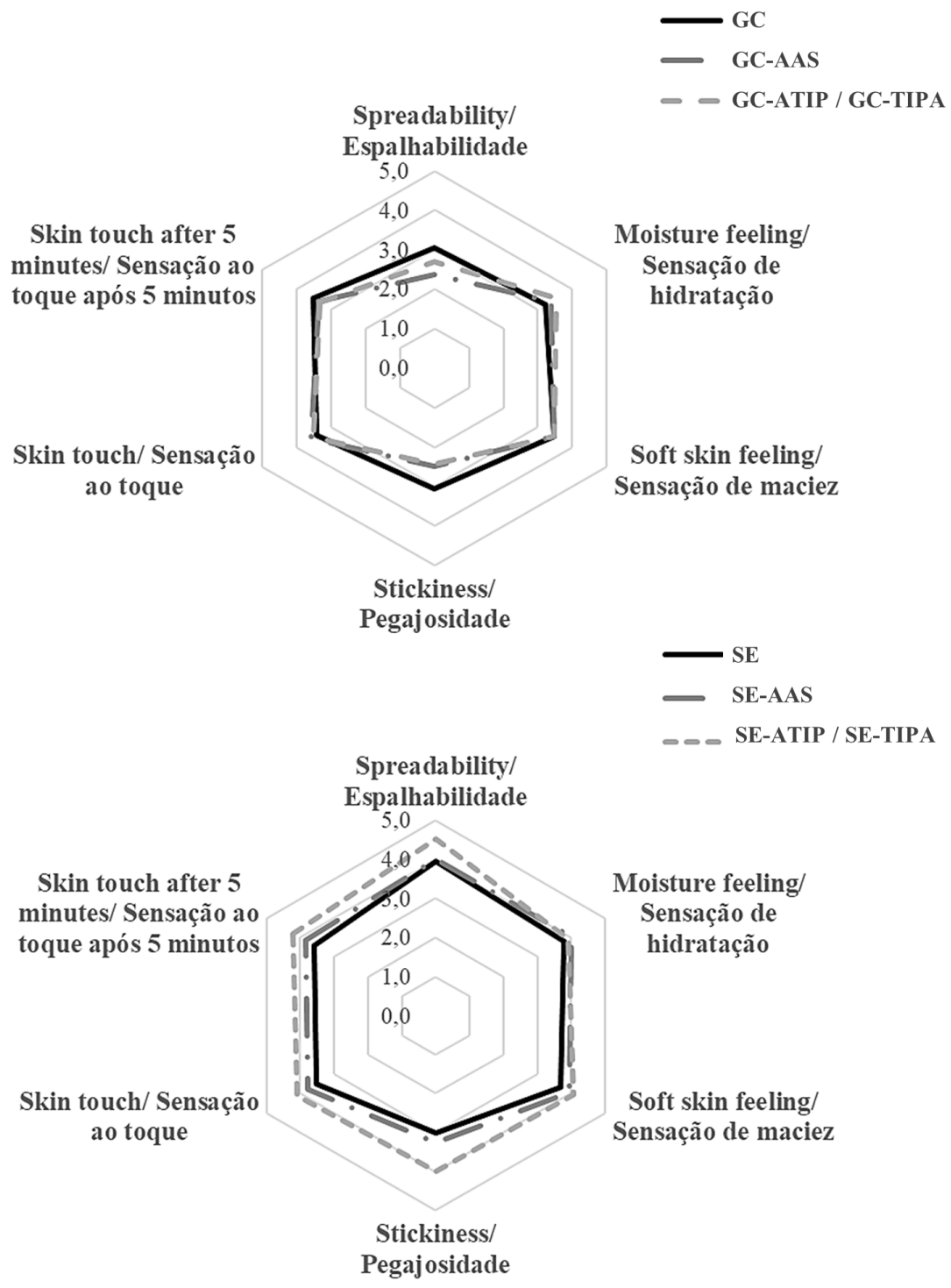

Figure 3 - Results of the sensory analysis of the studied formulations

Figura 3 - Resultados da avaliação sensorial das formulações estudadas 
higher values of cohesiveness and stickiness, parameters that most spoiled sensory perception, as they made it difficult to spread and absorb into the skin. Thus, the most viscous formulations had worse spreadability and stickiness, and this was consistent with other research findings (16).

Regarding clinical efficacy testing (Figures 4 and 5), an immediate moisturizing effect was observed on the forearm for all formulations, with higher values of stratum corneum water content for GC, GC-AAS and SEATIP. The control region and the malar region of the face remained unchanged. TEWL was reduced in the vez que SE e SE-TIPA apresentaram baixo trabalho de cisalhamento, indicativos de melhor espalhabilidade, de acordo com a correlação verificada entre os dois parâmetros $(10,15)$. Em contrapartida, os GC não tiveram boa aceitação, com intenção de compra entre 5 e $11 \%$. No teste de dureza, esses apresentaram valores superiores de coesividade e pegajosidade, parâmetros que mais prejudicaram a percepção sensorial, por dificultarem a espalhabilidade e absorção na pele. Com isso, confirmou-se que as formulações mais viscosas apresentaram pior espalhabilidade e pegajosidade, em concordância com outros trabalhos (16).
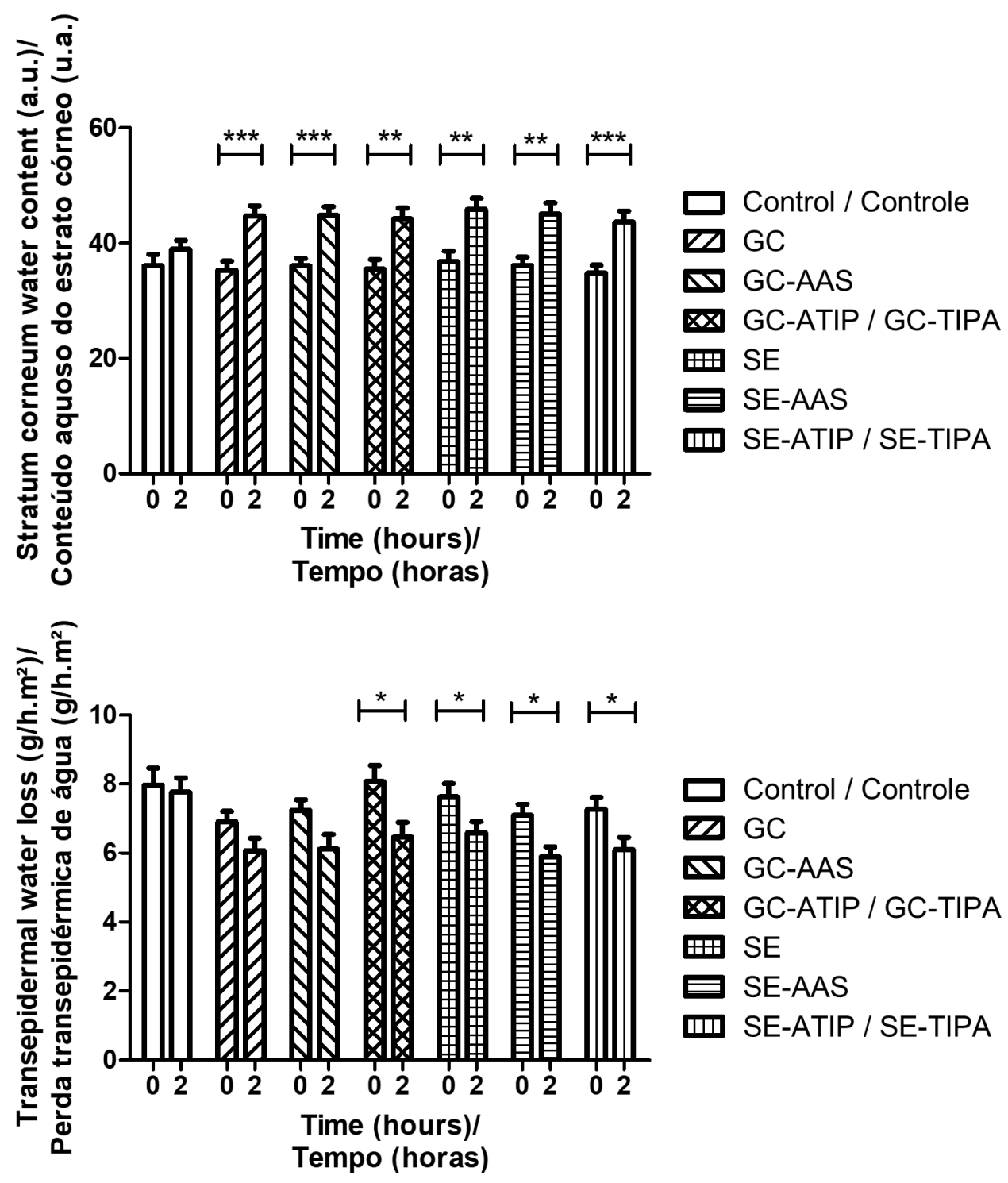

Figure 4 - Results of the clinical efficacy tests (hydration and TEWL) of the studied formulations on forearm areas. The measurements were made before any application (baseline or time 0 ) and after two hours of applying $20 \mu \mathrm{L}$ of each formulation in the areas for all nineteen subjects.

Figura 4 - Resultados dos testes clínicos de eficácia (hidratação e TEWL) das formulações estudadas nas áreas do antebraço. As medições foram feitas antes de qualquer aplicação (basal ou tempo 0) e após duas horas de aplicação de $20 \mu \mathrm{L}$ de cada formulação nas áreas para todas as dezenove participantes. 

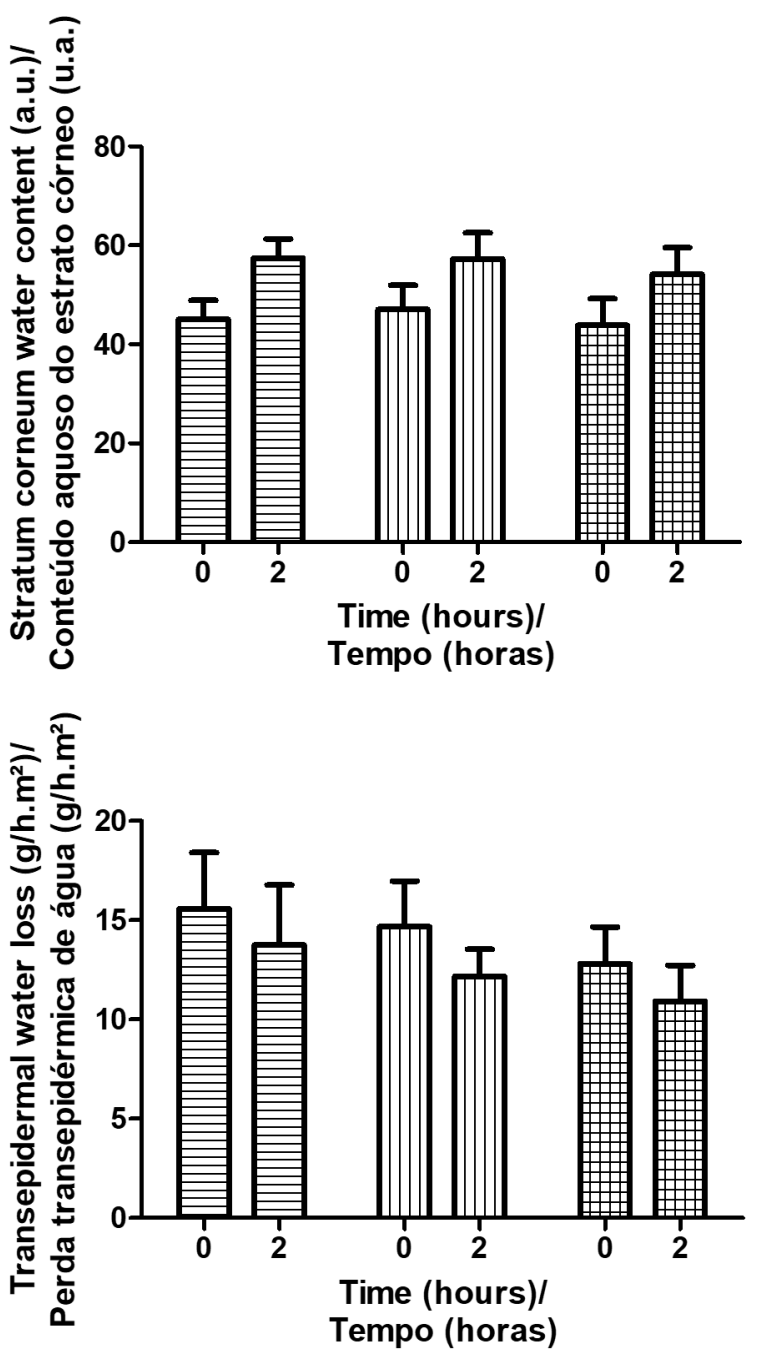

\author{
SE-AAS \\ 四 SE-ATIP / SE-TIPA \\ 曲 SE
}

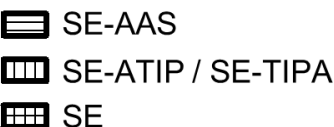

Figure 5 - Results of the clinical efficacy tests (hydration and TEWL) of the studied serums on malar area of the face. The measurements were made before any application (baseline or time 0 ) and after two hours of applying on the entire face $1 \mathrm{~g}$ of the designated formulation, according to the three groups. Figura 5 - Resultados dos testes clínicos de eficácia (hidratação e TEWL) dos séruns estudados na área malar do rosto. As medições foram feitas antes de qualquer aplicação (basal ou tempo 0) e após duas horas de aplicação no rosto todo de $1 \mathrm{~g}$ da formulação determinada, de acordo com os três grupos.

areas of the forearm in which GC-ATIP, SE, SE-AAS and SE-ATIP were applied, but no alterations were noted in the malar region. These different results between the two regions may have occurred due to physiological differences between the areas and also due to much lower basal hydration values in the forearms within the studied group, which could make moisturizing action more pronounced on these areas $(17,18)$. In skin microrelief analysis, no changes were observed in the parameters in any region, due to high interindividual variation. Finally, the serum formulations showed better efficacy, mainly on TEWL reduction.
Nos testes de eficácia clínica (Figuras 4 e 5), observouse efeito hidratante imediato para todas as formulações, com valores superiores de conteúdo aquoso do estrato córneo para GC, GC-AAS e SE-TIPA. A região controle e a região malar do rosto se mantiveram inalteradas. A TEWL reduziu nas áreas da pele do antebraço em que foram aplicadas GC-TIPA, SE, SE-AAS e SE-TIPA, mas sem alteração na região malar. A diferença de efeitos pode ter ocorrido devido às diferenças fisiológicas entre as regiões estudadas e pelos valores basais de hidratação dentro do grupo estudado terem sido muito inferiores na pele dos antebraços, fazendo com que a ação hidratante tenha sido mais pronunciada nessa região $(17,18)$. Na análise do microrrelevo cutâneo, não foram observadas alterações dos parâmetros 


\section{Conclusion}

Serum presented better stability and sensory perception, which correlated to physical-mechanical parameters, such as spreadability, and serum with ATIP were evaluated the best because it did not alter formulation's properties. The studied formulations were effective in the immediate hydration and only serums and gel cream containing ATIP reduced TEWL. In summary, serums were the cosmetic form that showed the greatest moisturizing efficacy and preference for study participants.

\section{Acknowledgments}

The authors thank CNPq and CNPq-PIBIC (Conselho Nacional de Desenvolvimento Científico e Tecnológico) for financial support and scholarships.

\section{Author Contributions}

G.B. performed the experimental part of the work and participated in the manuscript writing; G.C. assisted in the definition of the study protocol and in the writing of the manuscript. P.M.C. coordinated the study, defined the study protocol and evaluated and discussed results, supervising writing and approving the final version of the manuscript.

\section{Conflict of interest}

The senior editor co-authoring this manuscript had no participation in the review nor in the decision process.

All authors have declared there were no financial and/ or personal relationships that may present a potential conflict of interest. em nenhuma região estudada, em função da alta variação interindividual. Por fim, as formulações sérum mostraram melhor eficácia, especialmente na redução da TEWL.

\section{Conclusão}

O sérum apresentou melhor estabilidade e percepção sensorial, que foi correlacionada aos parâmetros físicomecânicos, como espalhabilidade, sendo o sérum com TIPA melhor avaliado, por não ter alterado as propriedades da formulação. As formulações estudadas foram eficazes na hidratação imediata e somente os séruns e o gel creme contendo TIPA reduziram a TEWL. Em síntese, os séruns foram a forma cosmética que apresentou maior eficácia hidratante e preferência pelos participantes do estudo.

\section{Agradecimentos}

Os autores agradecem ao CNPq e CNPq-PIBIC (Conselho Nacional de Desenvolvimento Científico e Tecnológico) pelo apoio financeiro e bolsas atribuidas.

\section{Contribuição dos Autores}

G.B. realizou a parte experimental do trabalho e participou na redação do manuscrito; G.C. auxiliou na definição do protocolo de estudo e na redação do manuscrito. P.M.C. é a coordenadora do estudo, definiu o protocolo de estudo, avaliou e discutiu os resultados e orientou a redação, aprovando a ultima versão do manuscrito.

\section{Conflito de interesses}

O editor senior envolvido na autoria deste manuscrito não teve qualquer participação no processo de revisão ou de decisão.

Todos os autores declararam não haver relações financeiras e/ou pessoais que possam representar um potencial conflito de interesses. 


\section{References / Referências}

1. Masaki H. Role of antioxidants in the skin: Anti-aging effects. Journal of Dermatological Science 2010; 58(2): 85-90.

2. Bissett, DL. Common cosmeceuticals. Clinics In Dermatology 2009; 27(5): 435-445.

3. Kim S, Lee TG. Stabilization of 1-ascorbic acid in cosmetic emulsions. Journal Of Industrial And Engineering Chemistry 2018 ; 57 : $193-198$.

4. Ochiai Y, et al. A new lipophilic pro-vitamin C, tetra-isopalmitoyl ascorbic acid (VC-IP), prevents UV-induced skin pigmentation through its anti-oxidative properties. Journal Of Dermatological Science 2006; 44(1): 37-44.

5. Zussman J, Ahdout J, Kim J. Vitamins and photoaging: Do scientific data support their use? Journal of the American Academy of Dermatology 2010; 63(3): 507-525.

6. Gallarate M, et al. On the stability of ascorbic acid in emulsified systems for topical and cosmetic use. International Journal of Pharmaceutics 1999; $188(2)$ : $233-241$.

7. Maia Campos PMBG, et al. Application of tetra-isopalmitoyl ascorbic acid in cosmetic formulations: Stability studies and in vivo efficacy. European Journal of Pharmaceutics and Biopharmaceutics 2012; 82: 580-586.

8. Shirata, MMF, PMBGM. Importância do perfil de textura e sensorial no desenvolvimento de formulações cosméticas. Surgical And Cosmetic Dermatology 2016; 8(3):223-230.

9. Surini S, Mubarak H, Ramadon D. Cosmetic Serum Containing Grape (Vitis vinifera L.) Seed Extract Phytosome: Formulation and in vitro Penetration Study. Jornal of Young Pharmacists 2018; 10(2): 51-55.

10. Calixto LS, Maia Campos PMBG. Physical Mechanical characterization of cosmetic formulations and correlation between instrumental measurements and sensorial properties. International Journal of Cosmetic Science 2017; 39: 527-534.

11. Borges CD, Vendruscolo CT. Xanthan Gum: characteristics and operational conditions of production. Semina: Ciências Biológicas e da Saúde 2008; 29(2): 171-188.

12. Maestro A, González C, Gutiérrez JM. Shear thinning and thixotropy of HMHEC and HEC water solutions. Journal of Rheology 2002; 46(6): $1445-1457$.

13. McMillan G, Montgomery R, Wang T. Opportunities for smart wireless pH, conductivity measurements. InTech Magazine (serial online) 2009 Dec [cited 2019 Jun 19]; 1(1): [8 screens]. in: URL: https:/www.isa.org/standards-and-publications/isa-publications/intech-magazine/2009/december/web-exclusiveopportunities-for-smart-wireless-ph-conductivity-measurements/

14. Gianeti MD, et al. Benefits of Combinations of Vitamin A, C and E Derivatives in the Stability of Cosmetic Formulations. Molecules 2012; 17(2): 22192230 .

15. Yilmaz E, Öğütcü M. Comparative analysis of olive oil organogels containing beeswax and sunflower wax with breakfast margarine. Journal of Food Science 2014; 79(9): 1732-1738.

16. Lukic M, et al. A combined approach in characterization of an effective w/o hand cream: the influence of emollient on textural, sensorial and in vivo skin performance. International Journal of Cosmetic Science 2012; 34(2): 140-149.

17. Paseto TR, Wagemaker TAL, Maia Campos PMBG. Immediate and long term effects of a cosmetic formulation with Cichorium intybus root extract. Biomedical and Biopharmaceutical Research 2017; 14(2): 220-232.

18. Tončić RJ, et al. Skin barrier and dry skin in the mature patient. Clinics In Dermatology 2018; 36(2): 109-115. 